\title{
Palliative care in intensive care units: why, where, what, who, when, how
}

\author{
Sebastiano Mercadante ${ }^{1}$, Cesare Gregoretti ${ }^{2}$ and Andrea Cortegiani ${ }^{2^{*}}$ (D)
}

\begin{abstract}
Palliative care is patient and family-centered care that optimizes quality of life by anticipating, preventing, and treating suffering when "curative" therapies are futile. In the Intensive Care Unit (ICU), critically ill patients receive life-sustaining therapies with the goal of restoring or maintaining organ function. Palliative Care in the ICU is a widely discussed topic and it is increasingly applied in clinics. It encompasses symptoms control and end-of-life management, communication with relatives and setting goals of care ensuring dignity in death and decision-making power. However, effective application of Palliative Care in ICU presupposes specific knowledge and training which anesthesiologists and critical care physicians may lack. Moreover, logistic issues such protocols for patients' selection, application models and triggers for consultation of external experts are still matter of debate.

The aim of this review is to provide the anesthesiologists and intensivists an overview of the aims, current evidence and practical advices about the application of palliative care in ICU.
\end{abstract}

Keywords: Palliative care, Intensive care unit, ICU, Patient-centered care, End-of-life care

\section{Background}

The aim of intensive care is the maintenance of vital functions to reduce mortality and prevent morbidity in patients with a severe critical illness. Despite the development of new technologies and the improvement of care, death rate in the intensive care unit (ICU) remains high $[1,2]$, ranging $20-35 \%$, with variations according geographical regions. Mortality rate was higher in upper-middle income countries than to ICUs in low and lower-middle or high-income [3]. In the latest years, ICU admissions in the last month of life have been growing up to $30 \%[1,4]$. When the organ dysfunction of critical illness does not respond to treatment, and the goals of care cannot be achieved anymore, or when life support becomes to be non proportional to expected prognosis, ICU physicians should provide an acceptable death $[5,6]$. When life-sustaining therapies are unable to meet the patient's goals, or paradoxically may result to be more burdensome than beneficial, withdrawal and withholding of therapies is a commonplace among ICU

\footnotetext{
*Correspondence: cortegiania@gmail.com

2Department of Biopathology and Medical Biotechnologies (DIBIMED). Section of Anestesia, Analgesia, Intensive Care and Emergency. Policlinico Paolo Giaccone, University of Palermo, Via del vespro 129, 90127 Palermo, Italy

Full list of author information is available at the end of the article
}

physicians [7]. In general, dying patients lack decision-making capacity. Advanced directives, when available, should guide the decision-making process, although it is often a medical team decision. This process may be complex and emotionally draining. Physician training in graduate and continuing medical education may provide guidance and support. Moreover, complications of polypharmacy, no realistic overview, poor attention for quality of life, and communication with relatives, are of concern. However, it is often difficult for physicians to initiate an appropriate discussion with patients' relatives. Thus, ICU clinicians require knowledge and competence on the many aspects of withholding/ withdrawing interventions and, in general, on end of life supports [7-11], including adoption of some treatment limiting the suffering, good communication with relatives, and how to afford some ethical issues. The use of sedatives, analgesics, and other non-pharmacological methods to ease distressing symptoms, as well as careful communication to support the decision-making process, including autonomy, capacity determination, and surrogacy, are of paramount importance, even during the phase of active treatments $[12,13]$. Moreover, ICU staying is also an unpleasant experience. Many symptoms commonly encountered in palliative care practice, such

(c) The Author(s). 2018 Open Access This article is distributed under the terms of the Creative Commons Attribution 4.0 International License (http://creativecommons.org/licenses/by/4.0/), which permits unrestricted use, distribution, and reproduction in any medium, provided you give appropriate credit to the original author(s) and the source, provide a link to the Creative Commons license, and indicate if changes were made. The Creative Commons Public Domain Dedication waiver (http://creativecommons.org/publicdomain/zero/1.0/) applies to the data made available in this article, unless otherwise stated. 
as pain, thirst, anxiety, sleep disturbances and dyspnea, frequently develop in critically ill patients [14-18]. These symptoms may persist even after ICU discharge and may produce a post-intensive care unit syndrome, with cognitive, psychiatric and physical consequences [1921]. Potentially, ICU clinicians should anticipate this approach to mitigate these problems [22]. Patients' families may experience psychological and physical distress, including depression, fear, anxiety, fatigue, anorexia, and early posttraumatic stress symptoms [23], and the persistence of symptoms after ICU can result in a "post-intensive care unit syndrome-family" in bereaved families [24-26]. Finally, ICU clinicians are at risk for emotional and psychological distress when facing these situations. Additional file 1 depicts critical care physician's doubts when a curative plan seems no longer effective.

Palliative care physicians are traditionally involved in issues regarding end of life care. Palliative care is patient and family-centered care with the aim of optimizing quality of life by anticipating, preventing, and treating suffering, providing a comprehensive management of patients facing incurable diseases, regardless of age, diagnosis, or prognosis. Moreover, palliative care focuses issues including symptom distress (physical, psychological, spiritual), capacity to communicate and share decision-making, while reducing the burden for caregivers $[9,27]$. Apparently, palliative care and intensive care may be the opposite ends of care; the former is considered as "talking medicine" and the latter as "technical medicine." However, there are commonalities between modalities of care, as both fields may overlap in virtuous circle to provide the best benefit for ICU patients. As a merger of approaches and cultures begin to seem natural, and collaborative opportunities are consequential, this review will examines some points regarding the apparent oxymoron existing between ICU and palliative care.

\section{Why}

Unrelieved and distressing symptoms are present for most ICU patients. In a prospective study of ICU patients at high risk of dying, distressful symptom prevalence ranged from 27 to $75 \%$. Delirium was found in about one-third of patients who could be evaluated [15]. Similarly, relatives showed a high level of symptom distress, with $57 \%$ having traumatic stress, $70-80 \%$ anxiety and depression, other than physical and emotional symptoms, particularly in the youngest [28]. These findings suggest that a palliative care assessment should be started as early as possible to allow more focused interventions to anticipate or minimize unnecessary suffering. In a retrospective study using medical record review and surveys of family members, it has been found that certain whole-person and preparation-for-death aspects of the dying process, and avoidance of aggressiveness at the end-of-life care, were more likely to be associated to better quality ratings. This finding suggests that care at the end of life in the ICU should not include managing pain and symptoms only, but also supporting death dignity and respect, which are typical issues necessitating palliative care experience [29]. It has been estimated that 14$20 \%$ of ICU patients meet the typical "triggers" for palliative care consultation [30]. Proactive palliative care involvement on ICU rounds for high-risk patients has been found to be associated with earlier ICU family meetings and shorter hospital length of stay [31]. Of interest, although few specific interventions were found to increase family satisfaction in ICU, good-quality communication, support for shared decision-making, and specific patient-care measures were associated with increased satisfaction with end-of-life care [32]. Thus, principles of palliative care need to be mandatorily applied in ICUs.

\section{Where}

Potentially, patients who are unfit for further aggressive treatments, could be transferred to a specialized palliative care setting, where there are physicians having certification in hospice and palliative medicine, and withdrawal procedures are commonly used in end-of-life care patients.. This modality has not been reported properly and is difficult to organize, also because hospice care is often offered in extra-hospital settings. Acute palliative care units may facilitate the process of transition of care. For instance, successful withdrawal of ventilatory support and a natural death at home were possible with a logistic support from the hospice organization and the expertise of the hospice team [33].

In a modern view, the principal domains of palliative care, including relief of distressing symptoms, effective communication about care goals, patient-focused decision-making, caregiver support, and continuity across care settings, should be performed in ICU [6, 9, 34].

\section{What}

Many issues are included in the content areas of palliative care. The management of physical and psychological symptoms, as well as spiritual and existential distress, prognostication, communication about care goals in relation to patient values and preferences are fundamental. Thus, a proactive identification of problems, an early sharing decision making with relatives, prospecting an advance care planning and possible scenarios for end of life decisions, are of paramount importance in ICU.

Moreover, ethical and legal aspects of decision making, transition planning, care during the dying process, and family support including grief and bereavement care complete the pattern of palliative care competencies. 
The goal-setting with a family experiencing high levels of distress or conflicts among family members, or supporting a bereaved family represent a typical examples of clinical changes [35].

Another relevant issue regards palliative sedation in the dying patients with high levels of terminal suffering. Some refractory symptoms may require to lower the level of consciousness by proportionate doses of hypnotics. Of interest, either the modality and the intent, are different from most sedation performed in ICU.

\section{Who}

Early efforts of palliative care in ICU focus on improving the quality of dying and death. However, its scope extends more broadly to meet the needs of patients affording life-supporting therapies and expected to survive. The general palliative care issues should be responsibility of all ICU clinicians, who need a basic knowledge and skills for symptoms management, appropriate techniques of communication, capability in sharing decision-making based on patients' values, goals and preferences (named also "integrative model") [34, 36]. Indeed, palliative care specialists can provide essential input in ICU. Although symptom management was the most common reason for palliative care consultation (named also "consultative model"), symptom assessment was infrequently documented. Furthermore, palliative care consultants performed better in offering spiritual support and managing documented symptoms [37]. Regrettably, the workforce of such specialists will remain inadequate to meet increasing needs in ICU population. For such reasons, it has been recommended a mixed model, where primary palliative care of ICU physicians is combined with specialist palliative care physicians.

\section{When}

Life-prolongation and palliation can be seen as dichotomous aspects of care. However, ICU clinicians and palliative care physicians both care critical and life-threatening conditions. Although the aims of palliative care and critical care may initially seem divergent, values and goals in critical care and palliative care are similar, as saving or prolonging life may conciliate with alleviating suffering and improving quality of life, and death. Of course, the primary goal of each discipline is the secondary goal of the other. One should consider that palliative care originated as end-of-life care in the 1960s [38]. However, since then its meaning and scope have expanded far beyond its roots. According to the World Health Organization [39], and a more recent and broader definition, the goal of palliative care is "to maintain and improve the quality of life of all patients and their families during any stage of life-threatening illness". Palliative care aims to prevent and relieve suffering by early identification, assessment, and treatment of physical and psychological symptoms, as well as emotional, and spiritual distress [39]. All patients receiving curative treatments should receive palliative care simultaneously and individually according to the patient's and family's needs and preferences [6]. Discussions about the changes of goals of care and a more proportional level of treatment should be started early in the ICU [40, 41]. During the course of ICU admission, frequent meetings with relatives are mandatory as the ICU patient's condition evolves. Life-support treatments should be systematically re-evaluated to determine if the care plan is achieving its goals. Physicians should facilitate these discussions, which should take place in a private and personal environment, involving patients' surrogates, and team members. Sharing opinion facilitates the decision-making process. Thus, palliative care assessment should be performed early during ICU admission, anytime typical issues for a palliative care evaluation emerge.

\section{How}

Basic symptom management and discussion of goals of care in relation to the patient's prognosis and preferences, are the core aspects of palliative care, and should be part of routine ICU practice, thus within the competency of any ICU clinician [8]. Decision-making on withholding and withdrawal of life-sustaining therapies in ICU is not homogeneous worldwide. This process depends on several factors such as legal, political, religious issues other than experience and patients' characteristics [42]. The capacity of withdrawal or withholding aggressive and futile treatments should belong to the armamentarium of any ICU clinician [43, 44]. For example, in withdrawal of mechanical ventilation, terminal extubation resulted to be a practice largely approved by family members, allowing relatives to be present at time of death, in comparison with terminal weaning. Of interest, no differences in length of stay and doses of sedatives were found [45].

End-of-life protocols seem to be effective in achieving adequate patient comfort in ICU. Few signs of distress were reported in most patients in whom life-sustaining measures were withdrawn. The use of opioids and sedatives increased significantly during treatment withdrawal but did not contribute to hasten death. Opioids are often successfully given to patients undergoing terminal withdrawal of mechanical ventilation $[10,13,46]$. Of interest, opioid doses were influenced by the level of previous opioid consumption (namely opioid-tolerance), and higher doses were associated with a longer time to death [47]. Thus, dying without distressing signs is practically possible and ethically feasible, as this does not hasten death $[48,49]$. 
There are two main models for ICU-palliative care integration. The "integrative model" seeks to embed palliative care principles and interventions into daily practice by the ICU team for all patients and families facing critical illness. In some circumstances an ICU physician might wish to obtain expert contributions from a palliative care team, as more advanced palliative care skills and interprofessional expert input may be necessary to face determined situations. The "consultative model" focuses on increasing the involvement of palliative care consultants in the care of ICUs patients identified as at highest risk for poor outcomes. This model provides and improves palliative care quality in the ICU. Of interest, other palliative care providers, including nurse practitioners, spiritual caregivers, and social workers could be involved. Psychological, social, and spiritual domains are relevant for the care of patients and their relatives.

The integration of palliative care experts in ICUs is of benefit to patients, families, and critical care clinicians. After palliative care consultation, $29 \%$ of patients were disconnected from mechanical ventilators, about $16 \%$ of patients discontinued the use of inotropic support, $15.3 \%$ stopped artificial nutrition, $6.4 \%$ stopped dialysis, and $2.5 \%$ discontinued hydration. Recommendations on pain and symptom management were made for $51 \%$ and $52 \%$ of patients. In this preliminary study there was an increase in the rate of the formalization of advance directives, with $33 \%$ of the patients having 'do not resuscitate' orders in place prior to consultation, and $83.4 \%$ after the intervention. Of interest, the involved team referred half of patients to hospice. Median survival times were not significantly different [50]. Preliminary evidence suggest that such models may be associated with improved quality of life, higher rates of formalization of advance directives and utilization of hospices, as well as lower use of certain non-beneficial life-prolonging therapies for patients who are at the end of life. Indeed, in the integrative model, palliative care principles are incorporated as part of routine practice by ICU physicians.

Each model can be successful in delivering palliative care in ICU, according to the resources and needs of individual ICUs [34]. Some points are in favour of the integrative model when workforce shortages may limit dependence on palliative care specialists, at least in the short term [30]. Moreover, reliance on specialty palliative care could undermine the therapeutic relationship with patients and relatives, giving the impression of fragmented care [51]. Finally, an external consultation may reduce the needs for ICU clinicians to develop palliative care knowledge and skills. On the other hand, specific situations often benefit from a palliative care specialist, when palliative care has not already been integrated among ICU physicians [9].
The mixed model includes both options [52]. The treating specialist could manage more relevant palliative care problems in combination with a palliative care specialist [35], as it occurs with other specialists to address peculiar problems arising in the clinical setting of critically ill patients. Criteria used to screen patients for unmet palliative care needs can trigger a palliative care consultation, while prompting care processes implemented by the ICU team itself [36]. Of interest, it has been reported that a palliative care consultation was associated with more frequent "do not resuscitate" code status and hospice referrals, hospital length of stay and direct cost reductions. Thus "trigger" programs in the ICU may improve utilization of palliative care services, improve patient comfort, and reduce invasive, often futile end-of-life care [53].

To meet palliative needs of critically ill patients and families, it will be relevant both to increase the capability of ICU clinicians to provide basic palliative care and to expand the specialist palliative care workforce. Clinical experience and a growing body of evidence suggest that palliative goals can improve the quality of care in critical ill patients [51]. Evidence regarding the use of palliative care in ICU, however, remains poor. Outcome measures were heterogeneous among study designs and many studies utilized different outcome measures, sometimes stratified between decedents and survivors. Because of the wide variation in outcome measures, study comparison was challenging. The heterogeneous study findings prevalently reported a decrease in ICU and hospital length of stay [51].

\section{Conclusion}

Although ICU and palliative care may seem to be polar opposites, they share fundamental features. In terms of evidence, ICU-based palliative care interventions are difficult to evaluate, due to the heterogeneity of studies. Existing data, however, suggest that proactive palliative care in the ICU, using both model of intervention, either consultative or integrative, may decrease hospital and ICU staying and do not affect mortality [31, 51]. Barriers to implement this approach include misperception of critical care and palliative care as sequential processes rather than complementary and simultaneous approaches, unrealistic expectations, and concerns that palliative care may hasten death. This misleading concepts are the consequence of insufficient training in communication and palliative care skills [52]. Indeed, a project framework and recommendations can be effectively used to increase the number of palliative care consults in the ICU and anticipate this kind of evaluation [54]. Daily pre-rounds between the palliative care and ICU teams increased palliative care services for patients at risk for poor outcomes, who may benefit from a 
palliative care consultation. Palliative care consultation increased significantly from 5 to $59 \%$ for patients who died in ICU during the intervention period [55].

ICU physicians should be competent in all aspects of terminal care, including the practical and ethical aspects of withdrawing different modalities of life-sustaining treatments and the use of pharmacological and non-pharmacologic approaches to limit the suffering of the dying process. A recent national survey with a large number of respondents suggested that training in palliative care and its clinical application need to be strongly implemented [56]. Many scientific organizations are encouraging the development of palliative care clinical programs locally in their hospitals and health care organizations $[7,57]$. ICU physicians should become familiar with such issues and ICU doctors should get a specific dedicated training in palliative care principles. Enhanced professional education and training in palliative care are the means for the necessary changes to ensure that all critically ill patients and their families have access to an excellent level of palliative care able to successfully meet their needs.

\section{Additional file}

Additional file 1: Critical care physician's most frequent questions about palliative care in ICU. The figure depicts critical care physician's doubts facing a clinical picture of a patient where the curative plan seems no longer effective. The questions describe most frequent open question about palliative care in ICU. (TIFF 4303 kb)

\section{Abbreviations}

ICU: Intensive Care Unit; PICS: Post-intensive care unit syndrome

\section{Acknowledgments}

We are glad to acknowledge Dr. Antonio Corrado, experienced anesthesiologist and illustrator, for providing the image in the Additional file.

\section{Availability of data and materials}

All data supporting this manuscript are available in the text.

\section{Authors' contributions}

SM, CG, AC conceived the content of this manuscript, draft the manuscript and approved the final version.

\section{Ethics approval and consent to participate}

Not applicable.

\section{Consent for publication}

Not applicable.

\section{Competing interests}

Dr. Sebastiano Mercadante is the Head of the Palliative Care and Cancer Pain Study Group of the Italian Society of Anesthesiology, Analgesia, Reanimation and Intensive Care (SIAARTI - Società Italiana di Anestesia, Analgesia, Rianimazione e Terapia Intensiva). Dr. Cesare Gregoretti declared to have no conflict of interest. Dr. Andrea Cortegiani is an Associate Editor for BMC Anesthesiology.

\section{Publisher's Note}

Springer Nature remains neutral with regard to jurisdictional claims in published maps and institutional affiliations.

\section{Author details}

Anesthesia and Intensive Care Unit and Pain Relief and Supportive-Palliative Care Unit, La Maddalena Cancer Center, Via san Lorenzo 312, 90145 Palermo, Italy. ${ }^{2}$ Department of Biopathology and Medical Biotechnologies (DIBIMED). Section of Anestesia, Analgesia, Intensive Care and Emergency. Policlinico Paolo Giaccone, University of Palermo, Via del vespro 129, 90127 Palermo, Italy.

Received: 10 February 2018 Accepted: 3 August 2018

Published online: 16 August 2018

\section{References}

1. Angus DC, Truog RD. Toward better ICU use at the end of life. JAMA. 2016; 315:255-6.

2. Angus DC, Barnato AE, Linde-Zwirble WT, Weissfeld LA, Watson RS, Rickert T, Rubenfeld GD. Use of intensive care at the end of life in the United States: an epidemiologic study. Crit Care Med. 2004;32:638-43.

3. Vincent J-L, Marshall JC, Namendys-Silva SA, François B, Martin-Loeches I, Lipman J, Reinhart K, Antonelli M, Pickkers P, Njimi H, Jimenez E, Sakr Y. Assessment of the worldwide burden of critical illness: the intensive care over nations (ICON) audit. Lancet Respir Med. 2014;2:380-6.

4. Teno JM, Gozalo PL, Bynum JPW, Leland NE, Miller SC, Morden NE, Scupp T, Goodman DC, Mor V. Change in end-of-life care for Medicare beneficiaries: site of death, place of care, and health care transitions in 2000, 2005, and 2009. JAMA. 2013;309:470-7.

5. Ho A, Tsai DF-C. Making good death more accessible: end-of-life care in the intensive care unit. Intensive Care Med. 2016;42:1258-60.

6. Cook D, Rocker G. Dying with dignity in the intensive care unit. N Engl J Med. 2014;370:2506-14.

7. Myburgh J, Abillama F, Chiumello D, Dobb G, Jacobe S, Kleinpell R, Koh Y, Martin C, Michalsen A, Pelosi P, Torra LB, Vincent J-L, Yeager S, Zimmerman $J$. End-of-life care in the intensive care unit: report from the task force of world Federation of Societies of intensive and critical care medicine. J Crit Care. 2016;34:125-30.

8. Edwards JD, Voigt LP, Nelson JE. Ten key points about ICU palliative care. Intensive Care Med. 2017:43:83-5.

9. Aslakson RA, Reinke LF, Cox C, Kross EK, Benzo RP, Curtis JR. Developing a research agenda for integrating palliative care into critical care and pulmonary practice to improve patient and family outcomes. J Palliat Med. 2017;20:329-43.

10. Downar J, Delaney JW, Hawryluck L, Kenny L. Guidelines for the withdrawal of life-sustaining measures. Intensive Care Med. 2016;42:1003-17.

11. Truog RD, Campbell ML, Curtis JR, Haas CE, Luce JM, Rubenfeld GD, Rushton $\mathrm{CH}$, Kaufman DC. Recommendations for end-of-life care in the intensive care unit: a consensus statement by the American college [corrected] of critical care medicine. Crit Care Med. 2008;36:953-63.

12. Mercadante $\mathrm{S}$, Giarratano A. The anesthesiologist and end-of-life care. Curr Opin Anaesthesiol. 2012:25:371-5.

13. Mercadante S, Giarratano A, Cortegiani A, Gregoretti C. Application of palliative ventilation: potential and clinical evidence in palliative care. Support Care Cancer. 2017;25:2035-9.

14. Choi J, Hoffman LA, Schulz R, Tate JA, Donahoe MP, Ren D, Given BA, Sherwood PR. Self-reported physical symptoms in intensive care unit (ICU) survivors: pilot exploration over four months post-ICU discharge. J Pain Symptom Manag. 2014;47:257-70.

15. Puntillo KA, Arai S, Cohen NH, Gropper MA, Neuhaus J, Paul SM, Miaskowski C. Symptoms experienced by intensive care unit patients at high risk of dying. Crit Care Med. 2010;38:2155-60.

16. Puntillo K, Nelson JE, Weissman D, Curtis R, Weiss S, Frontera J, Gabriel M, Hays R, Lustbader D, Mosenthal A, Mulkerin C, Ray D, Bassett R, Boss R, Brasel K, Campbell M. Palliative care in the ICU: relief of pain, dyspnea, and thirst--a report from the IPAL-ICU advisory board. Intensive Care Med. 2014; 40:235-48.

17. Mercadante S, Aielli F, Adile C, Valle A, Fusco F, Ferrera P, Caruselli A, Cartoni C, Marchetti P, Bellavia G, Cortegiani A, Masedu F, Valenti M, Porzio G. Epidemiology and characteristics of episodic breathlessness in advanced Cancer patients: an observational study. J Pain Symptom Manag. 2016;51:17-24.

18. Mercadante S, Aielli F, Adile C, Ferrera P, Valle A, Cartoni C, Pizzuto M, Caruselli A, Parsi R, Cortegiani A, Masedu F, Valenti M, Ficorella C, Porzio G. Sleep disturbances in patients with advanced Cancer in different palliative care settings. J Pain Symptom Manag. 2015;50:786-92. 
19. Desai SV, Law TJ, Needham DM. Long-term complications of critical care. Crit Care Med. 2011;39:371-9.

20. Saczynski JS, Marcantonio ER, Quach L, Fong TG, Gross A, Inouye SK, Jones RN. Cognitive trajectories after postoperative delirium. N Engl J Med. 2012; 367:30-9.

21. Pandharipande PP, Girard TD, Jackson JC, Morandi A, Thompson JL, Pun BT, Brummel NE, Hughes CG, Vasilevskis EE, Shintani AK, Moons KG, Geevarghese SK, Canonico A, Hopkins RO, Bernard GR, Dittus RS, Ely EW. Long-term cognitive impairment after critical illness. N Engl J Med. 2013, 369:1306-16.

22. Treece PD, Engelberg RA, Crowley L, Chan JD, Rubenfeld GD, Steinberg KP, Curtis JR. Evaluation of a standardized order form for the withdrawal of life support in the intensive care unit. Crit Care Med. 2004:32:1141-8.

23. Davidson JE, Aslakson RA, Long AC, Puntillo KA, Kross EK, Hart J, Cox CE, Wunsch H, Wickline MA, Nunnally ME, Netzer G, Kentish-Barnes N, Sprung CL, Hartog CS, Coombs M, Gerritsen RT, Hopkins RO, Franck LS, Skrobik Y, Kon AA, Scruth EA, Harvey MA, Lewis-Newby M, White DB, Swoboda SM, Cooke CR, Levy MM, Azoulay E, Curtis JR. Guidelines for family-centered Care in the Neonatal, pediatric, and adult ICU. Crit Care Med. 2017;45:103-28.

24. Davidson JE, Jones C, Bienvenu OJ. Family response to critical illness: postintensive care syndrome-family. Crit Care Med. 2012;40:618-24.

25. Schmidt M, Azoulay E. Having a loved one in the ICU: the forgotten family. Curr Opin Crit Care. 2012;18:540-7.

26. Choi J, Tate JA, Hoffman LA, Schulz R, Ren D, Donahoe MP, Given BA, Sherwood PR. Fatigue in family caregivers of adult intensive care unit survivors. J Pain Symptom Manag. 2014;48:353-63.

27. Byock I. Improving palliative care in intensive care units: identifying strategies and interventions that work. Crit Care Med. 2006;34:S302-5.

28. McAdam JL, Dracup KA, White DB, Fontaine DK, Puntillo KA. Symptom experiences of family members of intensive care unit patients at high risk for dying. Crit Care Med. 2010;38:1078-85.

29. Mularski RA, Heine CE, Osborne ML, Ganzini L, Curtis JR. Quality of dying in the ICU: ratings by family members. Chest. 2005;128:280-7.

30. Hua MS, Li G, Blinderman CD, Wunsch H. Estimates of the need for palliative care consultation across United States intensive care units using a triggerbased model. Am J Respir Crit Care Med. 2014;189:428-36.

31. Braus N, Campbell TC, Kwekkeboom KL, Ferguson S, Harvey C, Krupp AE, Lohmeier T, Repplinger MD, Westergaard RP, Jacobs EA, Roberts KF, Ehlenbach WJ. Prospective study of a proactive palliative care rounding intervention in a medical ICU. Intensive Care Med. 2016;42:54-62.

32. Hinkle $L$, Bosslet GT, Torke AM. Factors associated with family satisfaction with end-of-life care in the ICU: a systematic review. Chest. 2015;147:82-93.

33. Unger KM. Withdrawal of Ventilatory support at home on hospice. J Pain Symptom Manag. 2016;52:305-12.

34. Nelson JE, Bassett R, Boss RD, Brasel KJ, Campbell ML, Cortez TB, Curtis JR, Lustbader DR, Mulkerin C, Puntillo KA, Ray DE, Weissman DE. Models for structuring a clinical initiative to enhance palliative care in the intensive care unit: a report from the IPAL-ICU project (improving palliative care in the ICU). Crit Care Med. 2010;38:1765-72.

35. Quill TE, Abernethy AP. Generalist plus specialist palliative care--creating a more sustainable model. N Engl J Med. 2013;368:1173-5.

36. Nelson JE, Curtis JR, Mulkerin C, Campbell M, Lustbader DR, Mosenthal AC, Puntillo K, Ray DE, Bassett R, Boss RD, Brasel KJ, Frontera JA, Hays RM, Weissman DE. Choosing and using screening criteria for palliative care consultation in the ICU: a report from the improving palliative care in the ICU (IPAL-ICU) advisory board. Crit Care Med. 2013;41:2318-27.

37. Wysham NG, Hochman MJ, Wolf SP, Cox CE, Kamal AH. Performance of consultative palliative care model in achieving quality metrics in the ICU. J Pain Symptom Manag. 2016;52:873-7.

38. Saunders C. The evolution of palliative care. J R Soc Med. 2001;94:430-2.

39. WHO Definition of Palliative Care. http://www.who.int/cancer/palliative/ definition/en. Accessed 25 May 2018

40. Azoulay E, Chaize M, Kentish-Barnes N. Involvement of ICU families in decisions: fine-tuning the partnership. Ann Intensive Care. 2014:4:37.

41. Lilly CM, De Meo DL, Sonna LA, Haley KJ, Massaro AF, Wallace RF, Cody S. An intensive communication intervention for the critically ill. Am J Med. 2000;109:469-75.

42. Lobo SM, De Simoni FHB, Jakob SM, Estella A, Vadi S, Bluethgen A, MartinLoeches I, Sakr Y, Vincent J-L. Decision-making on withholding or withdrawing life support in the ICU: a worldwide perspective. Chest. 2017; 152:321-9.
43. Mark NM, Rayner SG, Lee NJ, Curtis JR. Global variability in withholding and withdrawal of life-sustaining treatment in the intensive care unit: a systematic review. Intensive Care Med. 2015;41:1572-85.

44. Connolly C, Miskolci O, Phelan D, Buggy DJ. End-of-life in the ICU: moving from "withdrawal of care" to a palliative care, patient-centred approach. Br J Anaesth. 2016;117:143-5.

45. Thellier D, Delannoy PY, Robineau O, Meybeck A, Boussekey N, Chiche A, Leroy $\mathrm{O}$, Georges $\mathrm{H}$. Comparison of terminal extubation and terminal weaning as mechanical ventilation withdrawal in ICU patients. Minerva Anestesiol. 2017:83:375-82.

46. Gregoretti C, Pisani L, Cortegiani A, Ranieri VM. Noninvasive ventilation in critically ill patients. Crit Care Clin. 2015;31:435-57.

47. Mazer MA, Alligood CM, Wu Q. The infusion of opioids during terminal withdrawal of mechanical ventilation in the medical intensive care unit. J Pain Symptom Manag. 2011;42:44-51.

48. Epker JL, Bakker J, Lingsma HF, Kompanje EJO. An observational study on a protocol for withdrawal of life-sustaining measures on two non-academic intensive care units in the Netherlands: few signs of distress, no suffering? J Pain Symptom Manag. 2015;50:676-84.

49. Kompanje EJO, van der Hoven B, Bakker J. Anticipation of distress after discontinuation of mechanical ventilation in the ICU at the end of life. Intensive Care Med. 2008:34:1593-9.

50. O'Mahony S, McHenry J, Blank AE, Snow D, Eti Karakas S, Santoro G, Selwyn P, Kvetan V. Preliminary report of the integration of a palliative care team into an intensive care unit. Palliat Med. 2010;24:154-65.

51. Aslakson R, Cheng J, Vollenweider D, Galusca D, Smith TJ, Pronovost PJ. Evidence-based palliative care in the intensive care unit: a systematic review of interventions. J Palliat Med. 2014;17:219-35.

52. Aslakson RA, Curtis JR, Nelson JE. The changing role of palliative care in the ICU. Crit Care Med. 2014;42:2418-28.

53. Hsu-Kim C, Friedman T, Gracely E, Gasperino J. Integrating palliative care into critical care: a quality improvement study. J Intensive Care Med. 2015; 30:358-64.

54. Sihra L, Harris M, O'Reardon C. Using the improving palliative care in the intensive care unit (IPAL-ICU) project to promote palliative care consultation. J Pain Symptom Manag. 2011;42:672-5.

55. Villarreal D, Restrepo Ml, Healy J, Howard B, Tidwell J, Ross J, Hartronft S, Jawad M, Sanchez-Reilly S, Reed K, Espinoza SE. A model for increasing palliative care in the intensive care unit: enhancing interprofessional consultation rates and communication. J Pain Symptom Manag. 2011;42: 676-9.

56. Cortegiani A, Russotto V, Raineri SM, Gregoretti C, Giarratano A, Mercadante S. Attitudes towards end-of-life issues in intensive care unit among Italian anesthesiologists: a nation-wide survey. Support Care Cancer. 2017;26: $1773-80$.

57. Lanken PN, Terry PB, Delisser HM, Fahy BF, Hansen-Flaschen J, Heffner JE, Levy M, Mularski RA, Osborne ML, Prendergast TJ, Rocker G, Sibbald WJ, Wilfond B, Yankaskas JR. An official American Thoracic Society clinical policy statement: palliative care for patients with respiratory diseases and critical illnesses. Am J Respir Crit Care Med. 2008:177:912-27.

Ready to submit your research? Choose BMC and benefit from:

- fast, convenient online submission

- thorough peer review by experienced researchers in your field

- rapid publication on acceptance

- support for research data, including large and complex data types

- gold Open Access which fosters wider collaboration and increased citations

- maximum visibility for your research: over $100 \mathrm{M}$ website views per year

At $\mathrm{BMC}$, research is always in progress.

Learn more biomedcentral.com/submissions 